Homology, Homotopy and Applications, vol.19(2), 2017, pp.131-139

\title{
A NOTE ON THICK SUBCATEGORIES AND WIDE SUBCATEGORIES
}

\author{
CHAO ZHANG AND HONGYAN CAI
}

\author{
(communicated by J. Daniel Christensen)
}

\begin{abstract}
For a triangulated category with a bounded t-structure, we prove that there is a bijection between wide subcategories of its heart and thick subcategories of the triangulated category which are closed under the corresponding cohomological functor. We prove that a finite-dimensional triangular algebra over an algebraically closed field is hereditary if and only if any thick subcategory of the bounded derived category is closed under the usual cohomological functor.
\end{abstract}

Dedicated to Professor Yingbo Zhang on the occasion of her 70th birthday.

\section{Introduction}

To study the structure of a given category, an important approach is classifying its subcategories with certain properties. In this context, the definitive work was due to Gabriel in 1960s [9]. For a triangulated category, thick subcategories are full triangulated subcategories closed under taking direct summands, which are used to gain information of the given triangulated category, and the classification of thick subcategories becomes an important theme in the study of triangulated categories, for instance, the derived categories of rings and stable module categories of self-injective algebras, see $[\mathbf{1 2}, \mathbf{1 9}, \mathbf{2 1}, \mathbf{3}, \mathbf{5}, \mathbf{4}, \mathbf{6}, \mathbf{7}, \mathbf{1 5}]$. On the level of abelian categories, especially for module categories of rings, a high emphasis has been placed on the wide subcategories, namely, the abelian subcategories closed under extensions. The concept of wide subcategory was first introduced by Hovey and he classified wide subcategories closed under small coproduct of the module categories for commutative noetherian rings [13] (see also [17]). Also for commutative noetherian rings, Takahashi introduced the concept of $H^{0}$-stable subcategory of derived category, and obtained a bijection between $H^{0}$-stable localizing subcategories in unbounded derived category and the wide subcategories of $\operatorname{Mod} A$ closed under small coproduct via the coherent subsets of the prime spectrum of $A[\mathbf{2 0}]$. Moreover, Brüning established the one-toone correspondence between the thick subcategories of bounded derived category of a hereditary abelian category and its wide subcategories [8].

This paper attempts to answer a natural question: which kinds of functors can connect the thick subcategories of a triangulated category and the wide subcategories of

Received July 18, 2016, revised December 15, 2016; published on October 18, 2017. 2010 Mathematics Subject Classification: 16E05, 16G20, 18E30.

Key words and phrases: wide subcategory, lattice isomorphism, hereditary algebra.

Article available at http://dx.doi.org/10.4310/HHA.2017.v19.n2.a8

Copyright (C) 2017, International Press. Permission to copy for private use granted. 
an abelian category? We find a partial answer in the following setting. Let $\mathcal{T}$ be a triangulated category with a bounded $t$-structure. One can define a cohomology functor $H^{0}$ from $\mathcal{T}$ to the heart $\mathcal{A}$ determined by the $t$-structure. The cohomology functor $H^{0}$ induces a bijection between the $H^{0}$-stable thick subcategories (the thick subcategories closed under $H^{0}$ ) of $\mathcal{T}$ and the wide subcategories of the abelian category $\mathcal{A}$. Indeed, we obtain the following theorem.

Theorem. Let $\mathcal{T}$ be a triangulated category with a bounded $t$-structure $(\mathcal{T} \leqslant 0, \mathcal{T} \geqslant 0)$. Then there is an isomorphism between the lattice of all $H^{0}$-stable thick subcategories of $\mathcal{T}$ and the lattice of all wide subcategories of $\mathcal{A}=\mathcal{T} \leqslant 0 \cap \mathcal{T} \geqslant 0$.

Let $A$ be an artin $R$-algebra over a commutative $\operatorname{artin} \operatorname{ring} R$. We denote by $\bmod A$ the category of finitely generated modules and $D^{b}(A)$ the bounded derived category of $\bmod A$. It is well known that $D^{b}(A)$ has a standard bounded $t$-structure whose heart is $\bmod A$ and cohomology functor is the usual cohomology functor. As a corollary, we classify the $H^{0}$-stable thick subcategories of $D^{b}(A)$ in terms of wide subcategories of $\bmod A$, which can be seen as Takahashi's result on the level of thick subcategories of bounded derived categories for artin algebras [20].

Corollary. Let $A$ be an artin R-algebra. Then there is a bijection between all $H^{0}$ stable thick subcategories of $D^{b}(A)$ and all wide subcategories of $\bmod A$.

The corollary above provides a classification of all the thick subcategories in the bounded derived category for those algebras satisfying the property $(\mathrm{H})$ : any thick subcategory of the bounded derived category is $H^{0}$-stable. The hereditary $\operatorname{artin} R$ algebras possess the property $(\mathrm{H})$ since any object in their bounded derived category is isomorphic to the direct sum of the all cohomologies with shifts, see Krause [16] for example. Therefore, the corollary can be seen as a generalization of Brüning's result for the module category of hereditary algebras [8, Theorem 5.1]. Moreover, we prove that a finite-dimensional triangular algebra $A$ over an algebraically closed field is hereditary if and only if $A$ satisfies the property $(\mathrm{H})$.

In the first section, we collect some properties of $t$-structures and prove the main theorem. The second section is then devoted to studying the $H^{0}$-stable thick subcategories of the bounded derived category.

\section{Acknowledgments}

The first author would like to thank Henning Krause, for his help and support during his visit in Bielefeld University. The authors are supported by the National Natural Science Foundation of China (Grant No. 11301105 and No. 11601098) and Natural Science Foundation of Guizhou Province (Grant No. QSF[2016]1038). Moreover, the authors are very grateful to the anonymous referees for many helpful comments.

\section{Bounded $t$-structures and $H^{0}$-stable thick subcategories}

Let $\mathcal{T}$ be a triangulated category with $\Sigma$ the shift functor. Form [2], a $t$-structure is a pair of isomorphism closed full subcategories $(\mathcal{T} \leqslant 0, \mathcal{T} \geqslant 0)$ satisfying

(1) $\mathcal{T} \leqslant 0$ is closed under $\Sigma$, and $\mathcal{T} \geqslant 0$ is closed under $\Sigma^{-1}$;

(2) $\operatorname{Hom}_{\mathcal{T}}\left(X, \Sigma^{-1} Y\right)=0$, for any $X \in \mathcal{T}^{\leqslant 0}, Y \in \mathcal{T}^{\geqslant 0}$; 
(3) For any $X \in \mathcal{T}$, we have a triangle

$$
X_{1} \rightarrow X \rightarrow \Sigma^{-1} X_{2} \rightarrow \Sigma X_{1}
$$

with $X_{1} \in \mathcal{T} \leqslant 0, X_{2} \in \mathcal{T} \geqslant 0$.

For any $n \in \mathbb{Z}$, set $\mathcal{T} \leqslant n=\Sigma^{-n} \mathcal{T} \leqslant 0, \mathcal{T} \geqslant n=\Sigma^{-n} \mathcal{T} \geqslant 0$. Recall from [10, Lemma 3.4.1], for any $t$-structure $(\mathcal{T} \leqslant 0, \mathcal{T} \geqslant 0)$, there exist two truncation functors $\tau_{\leqslant 0}: \mathcal{T} \rightarrow \mathcal{T} \leqslant 0$ and $\tau_{\geqslant 1}: \mathcal{T} \rightarrow \mathcal{T} \geqslant 1$, which are right adjoint and left adjoint to the inclusion functor respectively, such that for any object $X \in \mathcal{T}$, we have the distinguished triangle

$$
\tau_{\leqslant 0} X \rightarrow X \rightarrow \tau_{\geqslant 1} X \rightarrow \Sigma\left(\tau_{\leqslant 0} X\right) .
$$

More generally, we can define $\tau_{\leqslant n}=\Sigma^{-n} \circ \tau_{\leqslant 0} \circ \Sigma^{n}: \mathcal{T} \rightarrow \mathcal{T} \leqslant n$ and $\tau_{\geqslant n+1}=\Sigma^{-n} \circ$ $\tau_{\geqslant 1} \circ \Sigma^{n}: \mathcal{T} \rightarrow \mathcal{T}^{\geqslant n+1}$. Then for any object $X \in \mathcal{T}$ and $m \leqslant n$, there is an isomorphism

$$
\tau_{\leqslant n} \tau_{\geqslant m} X \cong \tau_{\geqslant m} \tau_{\leqslant n} X,
$$

which is defined to be $\tau_{[m, n]} X[\mathbf{1 0}$, Section 3.4.1(c)].

For any $t$-structure $(\mathcal{T} \leqslant 0, \mathcal{T} \geqslant 0)$, one defines the heart of the $t$-structure $\mathcal{A}=\mathcal{T} \leqslant 0 \cap$ $\mathcal{T} \geqslant 0$, which is an abelian category [10, Theorem 3.4]. Define the cohomology functor associated to $(\mathcal{T} \leqslant 0, \mathcal{T} \geqslant 0)$

$$
H^{0}=\tau_{[0,0]}: \mathcal{T} \rightarrow \mathcal{A}, \text { and } H^{n}=H^{0} \circ \Sigma^{n}: \mathcal{T} \rightarrow \mathcal{A}, n \in \mathbb{Z} .
$$

Remark 2.1. Let $\mathcal{A}$ be an abelian category and $D^{*}(\mathcal{A})$ be the derived category, where $* \in\{+,-, b, \emptyset\}$. Assume $\mathcal{D}^{\leqslant n}$ (resp. $\mathcal{D}^{\geqslant n}$ ) is the full subcategory of $D^{*}(\mathcal{A})$ consisting of all complexes with nonzero cohomologies concentrated in degree $i \leqslant n$ (resp. $i \geqslant n$ ). Then $\left(\mathcal{D}^{\leqslant 0}, \mathcal{D}^{\geqslant 0}\right)$ is the standard $t$-structure with the heart $\mathcal{A}$. Moreover, $H^{n}$ is the usual cohomology functor for any $n \in \mathbb{Z}$.

Recall that a $t$-structure $(\mathcal{T} \leqslant 0, \mathcal{T} \geqslant 0)$ of $\mathcal{T}$ is said to be bounded if $\cap_{n \in \mathbb{Z}} \mathcal{T} \leqslant n=$ $\cap_{n \in \mathbb{Z}} \mathcal{T}^{\geqslant n}=\{0\}$, and any $X \in \mathcal{T}$ satisfies $H^{i}(X)=0$ for almost all $i \in \mathbb{Z}[\mathbf{1 0}$, Definition 3.7.1]. Note that the definition coincides with the one given in [18], i.e., $\left(\mathcal{T}^{\leqslant 0}, \mathcal{T}^{\geqslant 0}\right)$ is bounded if for any $X \in \mathcal{T}$, there exists $m \leqslant n$ such that $X \in \mathcal{T} \leqslant n \cap$ $\mathcal{T} \geqslant m$.

Lemma 2.2. Let $\mathcal{T}$ be a triangulated category and $(\mathcal{T} \leqslant 0, \mathcal{T} \geqslant 0)$ be a bounded $t$-structure of $\mathcal{T}$ with $H^{0}$ the associated cohomology functor. Then

(1) $H^{0}$ is a cohomological functor.

(2) A morphism $f: X \rightarrow Y$ is an isomorphism if and only if $H^{i}(f)$ is isomorphism in $\mathcal{A}$ for all $i \in \mathbb{Z}$.

(3) $X \in \mathcal{T} \leqslant n$ if and only if $H^{i}(X)=0$ for any $i>n$. Similarly, $X \in \mathcal{T} \geqslant n$ if and only if $H^{i}(X)=0$ for any $i<n$.

(4) Any object $X \in \mathcal{T}$ can be generated by $\left\{H^{i}(X) \mid i \in \mathbb{Z}\right\}$ with triangles.

Proof. See [11, Chapter IV.11, Theorem] for the proof of (1)-(3) and we only prove the statement (4). Assume $X \in \mathcal{T}$, then there exist $m \leqslant n$ such that $X \in \mathcal{T} \leqslant n \cap \mathcal{T} \geqslant m$, and hence $H^{i}(X)=0$ for $i<m$ or $i>n$. Set

$$
h w(X)=\sup \left\{i-j+1 \mid H^{i}(X) \neq 0 \neq H^{j}(X)\right\}
$$

and we proceed by induction on $h w(X)$. If $h w(X)=1$, then the statement is trivial since $X$ is isomorphic to the nonzero cohomology by (2). If $h w(X)>1$, we assume 
$n=\max \left\{i \in \mathbb{Z} \mid H^{i}(X) \neq 0\right\}$, then we have the triangle

$$
\tau_{\leqslant n-1} X \rightarrow X \rightarrow \tau_{\geqslant n} X \rightarrow \Sigma\left(\tau_{\leqslant n-1} X\right) .
$$

Note that $h w\left(\tau_{\geqslant n} X\right)=1$ and $h w\left(\tau_{\leqslant n-1} X\right)=h w(X)-1$. Moreover, $\tau_{\geqslant n} X \cong H^{n}(X)$ by (1). Hence the statement follows by induction assumption.

The following definition is originally introduced in [20, Definition 2.5], and we generalize the usual cohomology functor to the cohomology functor determined by an arbitrary $t$-structure.

Definition 2.3. Suppose $\mathcal{T}$ is a triangulated category and $(\mathcal{T} \leqslant 0, \mathcal{T} \geqslant 0)$ is a $t$-structure of $\mathcal{T}$ with $H^{0}$ the associated cohomology functor. A thick subcategory $\mathcal{C}$ is $H^{0}$-stable if for any $X \in \mathcal{C}, H^{0}(X)$ lies in $\mathcal{C}$ under the inclusion $\mathcal{A} \hookrightarrow \mathcal{T}$.

Note that for any $H^{0}$-stable subcategory $\mathcal{C}$ and any $X \in \mathcal{C}$, any $H^{i}(X)$ lies in $\mathcal{C}$ since $\mathcal{C}$ is closed under shifts. Clearly, the intersection of two $H^{0}$-stable thick subcategories is also $H^{0}$-stable. Moreover, the collection of all $H^{0}$-stable thick subcategories forms a complete lattice, write $\mathcal{L}_{H s t}(\mathcal{T})$, in which the join of a collection of $H^{0}$ stable subcategories is the smallest one containing all of them and the meet is their intersection.

Now we recall the definition of wide subcategory of an abelian category in [13].

Definition 2.4. Let $\mathcal{A}$ be an abelian category. A full subcategory $\mathcal{M} \subseteq \mathcal{A}$ is a wide subcategory if it is closed under kernels, cokernels and extensions.

Evidently, any wide subcategory $\mathcal{M}$ is closed under taking direct summands. Indeed, if $M=M_{1} \oplus M_{2} \in \mathcal{M}$, then the kernel of $f: M \rightarrow M$ sending the element $\left(m_{1}, m_{2}\right)$ to $\left(0, m_{2}\right)$ is $M_{1}$. Since $\mathcal{M}$ is closed under kernels, it is also closed under direct summands. Note that the collection of wide subcategories of $\mathcal{A}$ is also a complete lattice and we denote it by $\mathcal{L}_{w}(\mathcal{A})$.

Theorem 2.5. Let $\mathcal{T}$ be a triangulated category with $(\mathcal{T} \leqslant 0, \mathcal{T} \geqslant 0)$ a bounded $t$-structure and the corresponding cohomological functor $H^{0}$. Then there is an isomorphism between the lattice $\mathcal{L}_{H s t}(\mathcal{T})$ of all $H^{0}$-stable thick subcategories of $\mathcal{T}$ and the lattice $\mathcal{L}_{w}(\mathcal{A})$ of all wide subcategories of the heart $\mathcal{A}=\mathcal{T} \leqslant 0 \cap \mathcal{T} \geqslant 0$ under the map

$$
\phi: \mathcal{C} \mapsto \text { the full subcategory with objects }\left\{H^{0}(X) \mid X \in \mathcal{C}\right\},
$$

with the inverse

$$
\varphi: \mathcal{M} \mapsto \text { the full subcategory with objects }\left\{X \in \mathcal{T} \mid H^{i}(X) \in \mathcal{M}, \forall i \in \mathbb{Z}\right\} \text {. }
$$

Proof. First we prove $\phi(\mathcal{C}) \in \mathcal{L}_{w}(\mathcal{A})$ for any $\mathcal{C} \in \mathcal{L}_{H s t}(\mathcal{T})$. Assume $M_{1}=H^{0}\left(X_{1}\right)$, $M_{2}=H^{0}\left(X_{2}\right)$ for $X_{1}, X_{2} \in \mathcal{C}$. Since $\mathcal{C}$ is a $H^{0}$-stable subcategory, $M_{1}, M_{2} \in \mathcal{C}$. If $f: M_{1} \rightarrow M_{2}$ is a morphism in $\phi(\mathcal{C})$, then there is a triangle in $\mathcal{C}$

$$
M_{1} \stackrel{f}{\longrightarrow} M_{2} \rightarrow M \rightarrow \Sigma M_{1},
$$

and we get an exact sequence in $\phi(\mathcal{C})$

$$
\cdots \rightarrow H^{-1}\left(M_{2}\right) \rightarrow H^{-1}(M) \rightarrow H^{0}\left(M_{1}\right) \stackrel{f}{\longrightarrow} H^{0}\left(M_{2}\right) \rightarrow H^{0}(M) \rightarrow H^{1}\left(M_{1}\right) \rightarrow \cdots
$$

with $H^{1}\left(M_{1}\right)=0=H^{-1}\left(M_{2}\right)$. Then $\operatorname{Ker}(f)=H^{-1}(M)$ and $\operatorname{Coker}(f)=H^{0}(M)$ lie in $\phi(\mathcal{C})$ since $\mathcal{C}$ is a $H^{0}$-stable. So $\phi(\mathcal{C})$ is closed under kernels and cokernels. Moreover, 
if there is a short exact sequence in $\mathcal{A}$ as follows

$$
0 \rightarrow M_{1} \stackrel{f}{\longrightarrow} M \stackrel{g}{\longrightarrow} M_{2} \rightarrow 0,
$$

then $f$ determines a triangle in $\mathcal{T}$

$$
M_{1} \stackrel{f}{\longrightarrow} M \rightarrow M_{2}^{\prime} \rightarrow \Sigma M_{1},
$$

which induces a long exact sequence in $\mathcal{A}$

$$
\cdots \rightarrow H^{-1}(M) \rightarrow H^{-1}\left(M_{2}^{\prime}\right) \rightarrow M_{1} \stackrel{f}{\rightarrow} M \rightarrow H^{0}\left(M_{2}^{\prime}\right) \rightarrow H^{1}\left(M_{1}\right) \rightarrow \cdots,
$$

with $H^{1}\left(M_{1}\right)=0=H^{-1}(M)$. Compare it with the short exact sequence $(*)$, we have $H^{0}\left(M_{2}^{\prime}\right) \cong M_{2}$ and $H^{i}\left(M_{2}^{\prime}\right)=0$ for $i \neq 0$. By Lemma $2.2(2), M_{2}^{\prime} \cong M_{2} \in \mathcal{C}$ and then $M \in \mathcal{C}$. Hence $M \in \phi(\mathcal{C})$ and $\phi(\mathcal{C})$ is a wide subcategory.

Next we will show the map $\varphi$ is well-defined. For any $\mathcal{M} \in \mathcal{L}_{w}(\mathcal{A}), \varphi(\mathcal{M})$ is closed under shifts by definition. Suppose there is a triangle

$$
X \rightarrow Y \rightarrow Z \rightarrow \Sigma X
$$

in $\mathcal{T}$ with $X, Z \in \varphi(\mathcal{M})$. Since $H^{0}$ is a cohomological functor by Lemma 2.2(1), we have a long exact sequence

$$
\cdots \rightarrow H^{n-1}(Z) \rightarrow H^{n}(X) \rightarrow H^{n}(Y) \rightarrow H^{n}(Z) \rightarrow H^{n+1}(X) \rightarrow \cdots
$$

satisfying $H^{i}(X), H^{i}(Z) \in \mathcal{M}$, for any $i \in \mathbb{Z}$. Since $\mathcal{M}$ is a wide subcategory, $H^{i}(Y) \in$ $\mathcal{M}$ for any $i \in \mathbb{Z}$. Thus $Y \in \varphi(\mathcal{M})$, and $\varphi(\mathcal{M})$ is closed under extensions. Note that $\mathcal{M}$ is closed under direct summands, then so is $\varphi(\mathcal{M})$, and thus $\varphi(\mathcal{M})$ is a thick subcategory. Moreover, for any object $X \in \varphi(\mathcal{M})$, we have $H^{0}(X) \in \mathcal{M}$ and hence, seen as an object in $\mathcal{T}, H^{0}(X) \in \varphi(\mathcal{M})$. Therefore, $\varphi(\mathcal{M}) \in \mathcal{L}_{H s t}(\mathcal{T})$.

To show $\varphi, \phi$ are lattice morphisms, we need to check:

(1) $\varphi\left(\mathcal{M}_{1} \vee \mathcal{M}_{2}\right)=\varphi\left(\mathcal{M}_{1}\right) \vee \varphi\left(\mathcal{M}_{2}\right)$ : Take $X \in \varphi\left(\mathcal{M}_{1} \vee \mathcal{M}_{2}\right)$, then $H^{i}(X) \in$ $\mathcal{M}_{1} \vee \mathcal{M}_{2}$ for any $i \in \mathbb{Z}$, i.e., $H^{i}(X)$ is generated by elements in $\mathcal{M}_{1}$ and $\mathcal{M}_{2}$ via taking kernels, cokernels and extensions. By the proof as above, we know kernels and cokernels in $\mathcal{M}_{1} \vee \mathcal{M}_{2}$ can be viewed as cohomologies of the object in $\varphi\left(\mathcal{M}_{1}\right) \vee \varphi\left(\mathcal{M}_{2}\right)$, and any exact sequence in $\mathcal{M}_{1} \vee \mathcal{M}_{2}$ can be lifted to be a triangle of object in $\varphi\left(\mathcal{M}_{1}\right) \vee \varphi\left(\mathcal{M}_{2}\right)$. Thus $H^{i}(X) \in \varphi\left(\mathcal{M}_{1}\right) \vee \varphi\left(\mathcal{M}_{2}\right)$, and then $X \in \varphi\left(\mathcal{M}_{1}\right) \vee \varphi\left(\mathcal{M}_{2}\right)$ since any object can be obtained by extensions with its cohomologies by Lemma 2.2(4). Conversely, $\varphi\left(\mathcal{M}_{i}\right) \subseteq \varphi\left(\mathcal{M}_{1} \vee \mathcal{M}_{2}\right)$ for $i=1,2$ and thus $\varphi\left(\mathcal{M}_{1}\right) \vee \varphi\left(\mathcal{M}_{2}\right) \subseteq \varphi\left(\mathcal{M}_{1} \vee\right.$ $\mathcal{M}_{2}$ ) by the definition of the join in $\mathcal{L}_{H s t}(\mathcal{T})$.

(2) $\varphi\left(\mathcal{M}_{1} \wedge \mathcal{M}_{2}\right)=\varphi\left(\mathcal{M}_{1}\right) \wedge \varphi\left(\mathcal{M}_{2}\right)$ : It is straightforward to check.

(3) $\phi\left(\mathcal{C}_{1} \vee \mathcal{C}_{2}\right)=\phi\left(\mathcal{C}_{1}\right) \vee \phi\left(\mathcal{C}_{2}\right)$ : Clearly $\phi\left(\mathcal{C}_{i}\right) \subseteq \phi\left(\mathcal{C}_{1} \vee \mathcal{C}_{2}\right)$ for $i=1,2$, and then $\phi\left(\mathcal{C}_{1}\right) \vee \phi\left(\mathcal{C}_{2}\right) \subseteq \phi\left(\mathcal{C}_{1} \vee \mathcal{C}_{2}\right)$. Conversely, for any $M \in \phi\left(\mathcal{C}_{1} \vee \mathcal{C}_{2}\right)$, we have $M=H^{0}(X)$ for some $X \in \mathcal{C}_{1} \vee \mathcal{C}_{2}$. Since $X$ can be obtained by the objects in $\mathcal{C}_{1}$ and $\mathcal{C}_{2}$ via taking cohomologies, taking direct summands, shifts and triangles, $M=H^{0}(X)$ can also be obtained by taking kernels, taking cokernels and extensions with the cohomologies of objects in $\phi\left(\mathcal{C}_{1}\right)$ and $\phi\left(\mathcal{C}_{2}\right)$, that is, $M \in \phi\left(\mathcal{C}_{1}\right) \vee \phi\left(\mathcal{C}_{2}\right)$.

(4) $\phi\left(\mathcal{C}_{1} \wedge \mathcal{C}_{2}\right)=\phi\left(\mathcal{C}_{1}\right) \wedge \phi\left(\mathcal{C}_{2}\right)$ : Trivial.

Now it remains to show $\varphi, \phi$ are mutually inverse. On one hand, it is clear we have $\phi \varphi(\mathcal{M}) \subseteq \mathcal{M}$. Since for any $M \in \mathcal{M}, M$ lies in $\varphi(\mathcal{M})$ seen as an object in $\mathcal{T}$, $\mathcal{M} \subseteq \phi \varphi(\mathcal{M})$. Hence $\mathcal{M}=\phi \varphi(\mathcal{M})$. On the other hand, $\mathcal{C} \subseteq \varphi \phi(\mathcal{C})$ and moreover, for 
any $X \in \varphi \phi(\mathcal{C})$, we have $H^{i}(X) \in \phi(\mathcal{C})$ for any $i \in \mathbb{Z}$, i.e., for each $i$, there is an object $Y_{i} \in \mathcal{C}$ such that $H^{i}(X)=H^{n_{i}}\left(Y_{i}\right)$ for some $n_{i}$. Then $H^{i}(X) \in \mathcal{C}$ since $\mathcal{C}$ is $H^{0}$-stable. Note that any object can be generated by the cohomologies with triangles by Lemma $2.2(4)$, then $X \in \mathcal{C}$ and hence $\varphi \phi(\mathcal{C})=\mathcal{C}$ follows.

\section{3. $H^{0}$-stable thick subcategories of bounded derived category}

Let $R$ be a commutative artin ring and $A$ be a connected associative artin $R$ algebra with identity. Throughout this section, we denote by $\bmod A$ the category of all right $A$-modules of finite length and by $K^{b}(\operatorname{proj} A)$ the homotopy category of bounded complexes of finitely generated projective $A$-modules. Moreover, $D^{b}(A)$ is the bounded derived category of $\bmod A$. For any $M \in \bmod A$, we denote by $S^{0} M$ the stalk complex with $M$ concentrated in degree 0 . In this section, we take the standard $t$-structure $\left(\mathcal{D}^{\leqslant 0}, \mathcal{D}^{\geqslant 0}\right)$ with $H^{0}$ the usual cohomology functor.

By the theorem in previous section, we obtain the following corollary which can be regarded as Takahashi's result within the context of thick subcategories of bounded derived categories for artin algebras [20].

Corollary 3.1. Let $A$ be an artin R-algebra. Then there is a bijection between $H^{0}$ stable thick subcategories of $D^{b}(A)$ and wide subcategories of $\bmod A$.

Note that the corollary above provides the classification of thick subcategories of bounded derived category for those algebras satisfying the property $(\mathrm{H})$ : any thick subcategory of the bounded derived category is $H^{0}$-stable. The following lemma implies that hereditary artin $R$-algebras satisfy the property $(\mathrm{H})$.

Lemma 3.2. (See Krause [16, Section 1.6]) Let $A$ be an artin $R$-algebra and $X^{\bullet} \in$ $D^{b}(A)$. Then $X^{\bullet} \cong \bigoplus_{i \in \mathbb{Z}} S^{0} H^{i}\left(X^{\bullet}\right)[-i]$. In particular, if $\mathcal{C} \subseteq D^{b}(A)$ is a thick subcategory and $X$ is an object in $\mathcal{C}$, then $S^{0} H^{i}\left(X^{\bullet}\right) \in \mathcal{C}$ for any $i \in \mathbb{Z}$.

Hence we have the following corollary, see also [8, Theorem 5.1].

Corollary 3.3. If $A$ is a hereditary artin $R$-algebra, then there is one-to-one correspondence between the thick subcategories of $D^{b}(A)$ and the wide subcategories of $\bmod A$.

By the above discussion, those algebras satisfying the property $(\mathrm{H})$ are interesting. We obtain a characterization of these triangular algebras. For this, we need the following preparations.

Lemma 3.4. Let $A$ be an artin $R$-algebra and $\mathcal{P}$ be the set of indecomposable projective $A$-modules. If $\mathcal{C}$ is a $H^{0}$-stable thick subcategory of $D^{b}(A)$ containing $S^{0} P$ for any $P \in \mathcal{P}$, then $\mathcal{C}=D^{b}(A)$. In particular, $D^{b}(A)$ is the smallest $H^{0}$-stable thick subcategory containing $K^{b}(\operatorname{proj} A)$.

Proof. For any $A$-module $M \in \bmod A$, we can take a minimal presentation $P^{-1} \stackrel{d}{\rightarrow} P^{0} \rightarrow M \rightarrow 0$. Let

$$
P^{\bullet}=\cdots \rightarrow 0 \rightarrow P^{-1} \stackrel{d}{\longrightarrow} P^{0} \rightarrow 0 \rightarrow \cdots,
$$

with $P^{0}$ concentrated in degree 0 . By assumption, $S^{0} P^{-1}, S^{0} P^{0} \in \mathcal{C}$, thus we have $P^{\bullet}=\operatorname{Cone}\left(S^{0} d\right) \in \mathcal{C}$. Since $\mathcal{C}$ is a $H^{0}$-stable thick subcategory, $S^{0} H^{0}\left(P^{\bullet}\right)=S^{0} M \in$ $\mathcal{C}$. Thus any complex $X^{\bullet} \in D^{b}(A)$ lies in $\mathcal{C}$. 
Remark 3.5. In the lemma, one can replace the set $\mathcal{P}$ with any set $\mathcal{X} \subseteq \bmod A$ satisfying that each $M \in \bmod A$ admits a surjection $X \rightarrow M$ or an injection $M \longmapsto X$ with $X \in \mathcal{X}$.

Proposition 3.6. Let $A$ be an artin $R$-algebra satisfying the property (H). Then

(1) $g l \cdot \operatorname{dim} A<\infty$;

(2) for any simple module $S$ with $\operatorname{pd} S>1$, if $\operatorname{Ext}_{A}^{1}(S, S)=0$ then $\operatorname{Hom}_{A}\left(S, \Omega^{2} S\right) \neq$ 0 , where $\Omega$ is the syzygy functor.

Proof. (1) If $g l . \operatorname{dim} A=\infty$, then the thick subcategory $K^{b}(\operatorname{proj} A)$ is not a $H^{0}$-stable thick subcategory by Lemma 3.4.

(2) Assume $S$ is a simple $A$-module satisfying that $\operatorname{pd} S>1$ and $\operatorname{Ext}_{A}^{1}(S, S)=0$, but $\operatorname{Hom}_{A}\left(S, \Omega^{2} S\right)=0$. We shall construct a thick subcategory which is not $H^{0}$ stable. Take a minimal presentation $P^{-1} \stackrel{d}{\rightarrow} P^{0} \rightarrow S \rightarrow 0$ and we consider the complex

$$
P^{\bullet}=\cdots \rightarrow 0 \rightarrow P^{-1} \stackrel{d}{\longrightarrow} P^{0} \rightarrow 0 \rightarrow \cdots,
$$

with $P^{0}$ concentrated in degree 0 .

First we will prove $\operatorname{Hom}_{D^{b}(A)}\left(P^{\bullet}, P^{\bullet}[i]\right)=0$ for any $i \neq 0$. Clearly for $i>1$ or $i<-1, \operatorname{Hom}_{D^{b}(A)}\left(P^{\bullet}, P^{\bullet}[i]\right)=0$. If $i=1$, then for any $f^{\bullet} \in \operatorname{Hom}_{D^{b}(A)}\left(P^{\bullet}, P^{\bullet}[1]\right)$, we have the following commutative diagram:

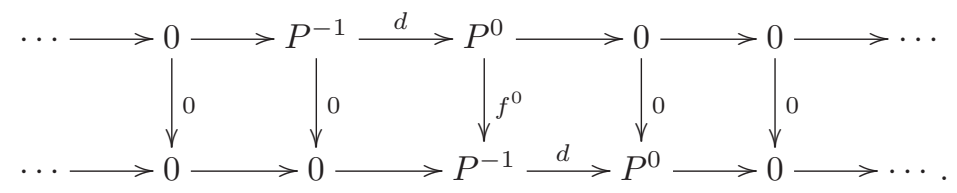

In particular, $f^{0} d=0=d f^{0}$. By the universal property of Ker $d=\Omega^{2} S, f^{0}$ factors through $\Omega^{2} S$, say $f^{0}=i f^{\prime}$, where $i$ is the canonical injection from $\Omega^{2} S$ to $P^{-1}$. Then $i f^{\prime} d=0$ implies $f^{\prime} d=0$ by the injectivity of $i$. Similarly by the universal property of Coker $d=S, f^{\prime}$ factors through $S$ and we write $f^{\prime}=f^{\prime \prime} \pi$. Note that $f^{\prime \prime} \in \operatorname{Hom}_{A}\left(S, \Omega^{2} S\right)=0$. Then $f^{\bullet}=0$. Now we consider the case $i=-1$. For any $f^{\bullet} \in \operatorname{Hom}_{D^{b}(A)}\left(P^{\bullet}, P^{\bullet}[-1]\right)$, we have the following commutative diagram:

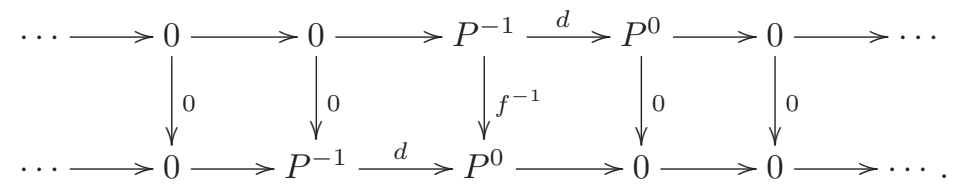

Since $\operatorname{Ext}_{A}^{1}(S, S)=0, P^{0}$ does not appear in $P^{-1}$ as a direct summand. Thus $f^{-1}$ factors through the inclusion $\Omega^{1} S=\operatorname{rad} P^{0} \hookrightarrow P^{0}$ and also factor through $d$ since $P^{-1}$ is the projective cover of $\Omega^{1} S$. Therefore, $f^{\bullet}=0$ in $\operatorname{Hom}_{D^{b}(A)}\left(P^{\bullet}, P^{\bullet}[-1]\right)$.

Next we will prove that $\mathcal{C}=\operatorname{add}\left\{P^{\bullet}[i] \mid i \in \mathbb{Z}\right\}$ is a thick subcategory of $D^{b}(A)$ which is not $H^{0}$-stable. First, $\operatorname{Hom}_{D^{b}(A)}\left(P^{\bullet}, P^{\bullet}\right) \cong \operatorname{Hom}_{K^{b}(A)}\left(P^{\bullet}, P^{\bullet}\right) \cong \underline{\operatorname{Hom}}_{A}(S, S)$ by $\left[\mathbf{1}\right.$, Chapter IV, Proposition 1.6], and $\operatorname{End}_{A}(S)$ is a division ring by Schur's Lemma, thus any nonzero element in $\operatorname{Hom}_{D^{b}(A)}\left(P^{\bullet}, P^{\bullet}\right)$ is an isomorphism. By the above discussion, $\operatorname{Hom}_{D^{b}(A)}\left(P^{\bullet}, P^{\bullet}[i]\right)=0$ for any $i \neq 0$ and any nonzero element in $\operatorname{Hom}_{D^{b}(A)}\left(P^{\bullet}, P^{\bullet}\right)$ is an isomorphism, and then $\mathcal{C}$ is a triangulated subcategory of $D^{b}(A)$. Moreover, $P^{\bullet}$ is indecomposable, see [22, Proposition 2] for instance, and thus 
$\mathcal{C}$ is closed under direct summands. So $\mathcal{C}$ is a thick subcategory of $D^{b}(A)$. Note that pd $S>1$ implies that $S=H^{0}\left(P^{\bullet}\right)$ is not in $\mathcal{C}$ viewed as a stalk complex. Therefore, the thick subcategory $\mathcal{C}$ is not a $H^{0}$-stable thick subcategory, which is a contradiction to the assumption.

Note that if the algebra is finite-dimensional over an algebraically closed field with finite global dimension, the no loop conjecture implies the ext-vanishing assumption [14]. The following theorem characterizes the finite-dimensional triangular algebras over algebraically closed fields satisfying the property $(\mathrm{H})$.

Theorem 3.7. Let $A$ be a finite-dimensional triangular algebra over an algebraically closed field, i.e., the ordinary quiver of $A$ contains no oriented cycles. Then $A$ is hereditary if and only if $A$ satisfies the property $(H)$.

Proof. If $A$ is a hereditary algebra, then the statement follows by Lemma 3.2. Conversely, suppose $A$ is a triangular algebra such that any thick subcategory of $D^{b}(A)$ is $H^{0}$-stable but not hereditary. Clearly, gl.dim $A<\infty$ and then $\operatorname{Ext}_{A}^{1}(S, S)=0$ for any simple modules $S$. Then by Proposition 3.6(2), there is a simple module $S_{0}$ with $\operatorname{pd} S_{0}>1$ and $\operatorname{Hom}_{A}\left(S_{0}, \Omega^{2} S_{0}\right) \neq 0$. Now we consider a minimal resolution of the simple module $S_{0}$,

$$
P^{\bullet}=\cdots \rightarrow P^{-m} \rightarrow \cdots \rightarrow P^{-2} \rightarrow P^{-1} \rightarrow P^{0} \rightarrow 0 .
$$

$\operatorname{Hom}_{A}\left(S_{0}, \Omega^{2} S_{0}\right) \neq 0$ implies that $S_{0}$ is isomorphic to a direct summand of the socle of $P^{-1}$. Then we have $\operatorname{Hom}_{A}\left(P^{0}, P^{-1}\right) \neq 0$ since $S_{0}$ is the isomorphic to the top of $P^{0}$. Thus, there exists an indecomposable direct summand $P$ of $P^{-1}$, which is not isomorphic to the indecomposable projective $A$-module $P^{0}$, such that $\operatorname{Hom}_{A}\left(P^{0}, P\right) \neq$ $0 \neq \operatorname{Hom}_{A}\left(P, P^{0}\right)$. It is a contradiction with the assumption that $A$ is triangular.

We conclude this paper with a question.

Question 3.8. Is there a similar characterization as Theorem 3.7 for general hereditary abelian categories?

\section{References}

[1] M. Auslander, I. Reiten, S.O. Smalø, Representation Theory of Artin Algebras, Cambridge University Press, 1997.

[2] A. Beilinson, J. Bernstein, P. Deligne, Faisceaux Pervers, Astérisque 100 (1982).

[3] D.J. Benson, J.F. Carlson, J. Rickard, Thick subcategories of the stable module category, Fund. Math. 153 (1997), 59-80.

[4] D. Benson, S.B. Iyengar, H. Krause, Local cohomology and support for triangulated categories, Ann. Sci. Éc. Norm. Supér. 41 (2008), 573-619.

[5] D.J. Benson, S.B. Iyengar, H. Krause, Stratifying modular representations of finite groups, Ann. of Math. 174 (2011), 1643-1684.

[6] D. Benson, S.B. Iyengar, H. Krause, Stratifying triangulated categories, J. Topol. 4 (2011), 641-666.

[7] D. Benson, S.B. Iyengar and H. Krause, A local-global principle for small triangulated categories, Math. Proc. Camb. Philos. Soc. 158 (2015), 451-476. 
[8] K. Brüning, Thick subcategories of the derived category of a hereditary algebra, Homology Homotopy Appl. 9 (2007), 165-176.

[9] P. Gabriel, Des catégories abéliennes, Bull. Soc. Math. France 90 (1962), 323448.

[10] S.I. Gelfand, Yu.I. Manin, Homological Algebra, Springer-Verlag, Berlin, 1999.

[11] S.I. Gelfand, Yu.I. Manin, Methods of Homological Algebra, second ed., Springer-Verlag, Berlin, 2003.

[12] M.J. Hopkins, Global methods in homotopy theory, in: Homotopy Theory, Durham, 1985, in: London Mathematical Society Lecture Note Series, vol. 117, Cambridge Univ. Press, Cambridge, 1987, 73-96.

[13] M. Hovey, Classifying subcategories of modules, Trans. Amer. Math. Soc. 353 (2001), 3181-3191.

[14] K. Igusa, Notes on the no loops conjecture, J. Pure Appl. Algebra 69 (1990), $161-176$

[15] C. Köhler, Thick subcategories of finite algebraic triangulated categories, arXiv:1010.0146.

[16] H. Krause, Derived categories, resolutions, and Brown representability, Contemp. Math. 436 (2007), 101-139.

[17] H. Krause, Thick subcategories of modules over commutative Noetherian rings (with an appendix by Srikanth Iyengar), Math. Ann. 340 (2008), 733-747.

[18] J. Le and X.W. Chen, Karoubianness of a triangulated category, J. Algebra, 310 (2007), 452-457.

[19] A. Neeman, The chromatic tower for $\mathrm{D}(\mathrm{R})$, with an appendix by Marcel Bökstedt, Topology 31 (3) (1992), 519-532.

[20] R. Takahashi, On localizing subcategories of derived categories, J. Math. Kyoto Univ. 49 (4) (2009), 771-783.

[21] R.W. Thomason, The classification of triangulated subcategories, Compos. Math. 105 (1997), 1-27.

[22] C. Zhang, Y. Han, Brauer-Thrall type theorems for derived module categories, Algebr. Represent. Theor. 19 (2016), 1369-1386.

Chao Zhang zhangc@amss.ac.cn

Department of Mathematics, Guizhou University, Guiyang 550025, P.R. China

Hongyan Cai sally-chy@163.com

Department of Mathematics, Guizhou University, Guiyang 550025, P.R. China 